[11] I. Tomioka, M. Hyozo, M. Okabe, S. Kishida, T. Arakawa, and Y. Kuramitsu, "Current control buffer for multi switching CMOS SOG," in Proc. IEEE Custom Integrated Circuits Conf., 1990, pp. 11.7.1-11.7.4.

[12] K. Furutani, H. Miyamoto, Y. Morooka, M. Suwa, and H. Ozaki, "Adjustable output driver with a self-recovering $V_{\mathrm{pp}}$ generator for a 4 M × 16 DRAM," IEEE J. Solid-State Circuits, vol. 29, pp. 308-310, Mar. 1994.

[13] M. H. Ku, “Adaptive output driver," M.Phil. thesis, Chinese Univ. Hong Kong, 1995.

[14] C. S. Choy, C. F. Chan, and M. H. Ku, "Feedback control circuit design technique to suppress power noise in high speed output driver," in Proc. Int. Symp. Circuits Systems, Apr. 1995, pp. 307-310.

[15] C. S. Choy, C. F. Chan, and M. H. Ku, "Comprehensive power bus noise analysis," in Proc. Int. Midwest Symp. Circuits Systems, Aug. 1995, pp. 1220-1223.

\section{Identification and Control of Unknown Chaotic Systems via Dynamic Neural Networks}

\author{
A. S. Poznyak, Wen Yu, and E. N. Sanchez
}

\begin{abstract}
Identification and control problems for unknown chaotic dynamical systems are considered. Our aim is to regulate the unknown chaos to a fixed point or a stable periodic orbit. This is realized by following two contributions. First, a dynamic neural network is used as identifier. The weights of the neural networks are adjusted by the sliding mode technique. Second, we derive a local optimal controller via the neuroidentifier to remove the chaos in a system. The identification error and trajectory error are guaranteed to be bounded. The controller proposed in this paper is effective for many chaotic systems, including the Lorenz system, Duffing equation, and Chua's circuit.
\end{abstract}

Index Terms - Chaos, neural networks, identification, sliding mode control, learning.

\section{INTRODUCTION}

Control chaos is one of the topics gaining great importance and attention in physics and engineering publications. Although the model descriptions of some chaotic systems are simple, the dynamic behaviors are complex. Recently, many researchers have managed to use modern elegant theories to control chaotic systems, most of them based on the exact chaotic model (differential equations). Linear state feedback [2] is very simple and easily implemented for the nonlinear chaotic systems. The Lyapunov-type method [5] is a more general synthesis approach for nonlinear controller design. The feedback linearization [3] technique is an effective nonlinear geometric theory for nonlinear chaos control. If the chaotic system is partly known, for example the differential equation is known but some of the parameters are unknown, adaptive control methods [10] are required.

In general, the unknown chaos is a black box belonging to a given class of nonlinearities. Therefore, a nonmodel-based method is suitable. The neurocontroller is popular for controlling unknown

Manuscript received March 24, 1998; revised October 28, 1998 and January 25,1999 . This was supported in part by CONACYT, Mexico, under Grants 0652-A9506 and 28070A. This paper was recommended by Associate Editor D. Liu.

A. S. Poznyak and W. Yu are with the Seccion de Control Automatico, CINVESTAV-IPN, 07000, Mexico.

E. N. Sanchez is with the CINVESTAV, Unidad Guadalajara, Guadalajara, 44550, Mexico.

Publisher Item Identifier S 1057-7122(99)09752-4. chaotic system. Yeap and Ahmed [9] used multilayer perceptrons to control chaotic systems. Chen and Dong [2] suggested direct and indirect neurocontrollers for chaos. Both of them were based on inverse modeling, i.e., neural networks are applied to learn the inverse dynamics of the chaotic systems. There are some drawbacks to this kind of technique, suchas lack of robustness, the demand of persistent excitation for the input signal and the non-one-to-one mapping of the inverse model. Another approach is identifier-based control. Two types of neural networks can be applied to identify dynamic systems with chaotic trajectories. The static neural network connected with a dynamic linear model is used to approximate a chaotic system [2], but the computing time is very long and some a priori knowledge of chaotic systems is needed. The dynamic neural networks can minimize the approximation error of the chaotic behavior [6], however, the number of neurons and the value of their weights are not determined. It is difficult to identify chaotic systems off line via neural networks, because the dynamic of chaos are very fast. From a practical point of view, the existing results are not satisfactory for the controller design.

One main point of this paper is to apply the sliding mode technique to the weights learning of dynamic neural networks. This approach can overcome the shortages of chaos identification. To the best of our knowledge, the sliding mode technique scarcely has been used in neural network weights learning [6]. We prove that the identification error converges to a bounded zone by means of a Lyapunov function technique. A local optimal controller [4] is used to compensate the modeling error. The effectiveness of the suggested approach is illustrated by its implementation to the Lorenz system, Duffing equation and Chua's circuit.

\section{Neuro Identifier with SLIDING Mode Learning}

A general nonlinear system can be described as follows:

$$
\dot{x}_{t}=f\left(x_{t}, u_{t}, t\right)
$$

where $x_{t} \in \Re^{n}$ is the system state vector at time $t \in R^{+}:=\{t: t \geq$ $0\} ; u_{t} \in \Re^{n}$ is a given control action; and $f(\cdot): \Re^{n+n+1} \rightarrow \Re^{n}$ is an unknown nonlinear function describing the dynamics of this system and belonging to a given class $\Phi$ of nonlinear functions. We say that a control $u_{t}$ is admissable if for any initial conditions and any nonlinearity from $\Phi$, it is piecewise continuous and it provides a piecewise differentiable solution $x_{t}$ which satisfies the following piecewise continuity assumption:

A1:

$$
\begin{aligned}
\left\|\dot{x}_{s}-\dot{x}_{t}\right\| & =\left\|f\left(x_{s}, u_{s}, s\right)-f\left(x_{t}, u_{t}, t\right)\right\| \\
& \leq C_{\tau}+D_{\tau}|s-t|
\end{aligned}
$$

for any $s, t \in R^{+}(|s-t| \leq \tau)\left(C_{\tau}\right.$ and $D_{\tau}$ are known nonnegative constants). Notice that for the chaotic systems given by known differential equations with a continuous right-hand side (it is necessary for the existence of a corresponding solution), this property is valid.

The main aim is to design an online fast identifier for the class of unknown chaotic systems and to realize control via this neuroidentifier. Let us consider the following dynamic neural network:

$$
\dot{\hat{x}}_{t}=A \hat{x}_{t}+W_{1, t} \sigma\left(V_{1, t} \hat{x}_{t}\right)+W_{2, t} \phi\left(V_{2, t} \hat{x}_{t}\right) u_{t}
$$

where $\hat{x}_{t} \in \Re^{n}, \sigma: \Re^{p} \rightarrow \Re^{k}, A \in \Re^{n \times n}$ is a Hurtwitz matrix, $W_{1, t} \in \Re^{n \times k}, V_{1, t} \in \Re^{p \times n}, W_{2, t} \in \Re^{n \times n}, V_{2, t} \in \Re^{q \times n}, \phi(\cdot)$ is $\Re^{n \times n}$ diagonal matrix, i.e., $\phi(\cdot)=\operatorname{diag}\left[\phi\left(V_{2, t} \hat{x}_{t}\right)_{1} \cdots \phi\left(V_{2, t} \hat{x}_{t}\right)_{n}\right]$. 
Here $\hat{x}_{t}$ is the state of the neural network, $W_{1, t}, W_{2, t}$ and $V_{1, t}, V_{2, t}$ are the weight matrixes describing the output and hidden layers connections, respectively, and $\sigma(\cdot)$ and $\phi(\cdot)$ are sigmoidal vector functions responsible for nonlinear state feedback. The most simple structure without any hidden layers (containing only the input and output layers) corresponds to the case when

$$
p=q=n \quad \text { and } \quad V_{1}=V_{2}=I .
$$

To simplify our further analysis, following to [7] and [6], To simplify our further analysis, we will use the following dynamic neural networks [6], [7]:

$$
\dot{\hat{x}}_{t}=A \hat{x}_{t}+W_{1, t} \sigma\left(\hat{x}_{t}\right)+W_{2, t} \phi\left(\hat{x}_{t}\right) u_{t} .
$$

Let us consider an admissable control $u_{t}$ and define the corresponding identification error as $\Delta_{t}:=x_{t}-\hat{x}_{t}$. From (1) and (5) we derive

$$
\begin{aligned}
\dot{\Delta}_{t}= & \dot{x}_{t}-\dot{\hat{x}}_{t}=f\left(x_{t}, u_{t}, t\right)-A \hat{x}_{t}-W_{1, t} \sigma\left(\hat{x}_{t}\right) \\
& -W_{2, t} \phi\left(\hat{x}_{t}\right) u_{t} .
\end{aligned}
$$

Because $f\left(x_{t}, u_{t}, t\right)$ is unknown (we only assume that $f \in \Phi$ ), we will use the following approximation:

$$
f\left(x_{t}, u_{t}, t\right)=\frac{x_{t}-x_{t-\tau}}{\tau}+\delta_{t}
$$

for a small enough $\tau \in R^{+}$. The vector $\delta_{t}$ is the approximation error at time $t$. In view of (1), its norm can be estimated as

$$
\begin{aligned}
\left\|\delta_{t}\right\| & =\left\|\tau^{-1}\left(x_{t}-x_{t-\tau}\right)-f\left(x_{t}, u_{t}, t\right)\right\| \\
& =\left\|\tau^{-1} \int_{t-\tau}^{t} \dot{x}_{s} d s-f\left(x_{t}, u_{t}, t\right)\right\| \\
& =\left\|\tau^{-1}\left[\int_{t-\tau}^{t} f\left(x_{s}, u_{s}, s\right)-f\left(x_{t}, u_{t}, t\right)\right] d s\right\| \\
& \leq \tau^{-1} \int_{t-\tau}^{t}\left\|f\left(x_{s}, u_{s}, s\right)-f\left(x_{t}, u_{t}, t\right)\right\| d s .
\end{aligned}
$$

Using $\mathbf{A} 1$ we derive that for any $t \geq 0$

$$
\left\|\delta_{t}\right\| \leq C_{\tau}+\frac{\tau}{2} D_{\tau} .
$$

After substituting (7) into (6), we conclude that

$$
\begin{gathered}
\dot{\Delta}_{t}=\frac{x_{t}-x_{t-\tau}}{\tau}-A \hat{x}_{t}-\left[\begin{array}{ll}
W_{1, t} & W_{2, t}
\end{array}\right] \\
\cdot\left[\begin{array}{c}
\sigma\left(\hat{x}_{t}\right) \\
\phi\left(\hat{x}_{t}\right) u_{t}
\end{array}\right]+\delta_{t}
\end{gathered}
$$

In order to guarantee the sliding mode behavior (see [8] and [6]), the following relation must be satisfied:

$$
\begin{aligned}
-P \operatorname{sign}\left(\Delta_{t}\right)= & \frac{x_{t}-x_{t-\tau}}{\tau}-A \hat{x}_{t}-\left[\begin{array}{ll}
W_{1, t} & W_{2, t}
\end{array}\right] \\
& \cdot\left[\begin{array}{c}
\sigma\left(\hat{x}_{t}\right) \\
\phi\left(\hat{x}_{t}\right) u_{t}
\end{array}\right]
\end{aligned}
$$

to obtain the following sliding mode dynamics:

$$
\dot{\Delta}_{t}=-P \operatorname{sign}\left(\Delta_{t}\right)+\delta_{t}
$$

where $P=\operatorname{diag}\left[P_{1} \cdots P_{n}\right]$ is positive diagonal matrix $\operatorname{sign}\left(\Delta_{t}\right):=$ $\left(\operatorname{sign}\left(\Delta_{1, t}\right), \cdots, \operatorname{sign}\left(\Delta_{n, t}\right)\right)^{T}$. Although the right-hand side of (11) is discontinuous, according to Filippov's conditions (see [8]), the existence of an almost everywhere piecewise differentiable solution can be guaranteed.
Remark 1: One of the possible weight selections $\left[W_{1, t} W_{2, t}\right]$ to fulfil (10) (perhaps excepting a subspace of a smaller dimension) is the pseudoinverse matrix given by

$$
\begin{aligned}
& {\left[\hat{W}_{1, t} \quad \hat{W}_{2, t}\right]=\left[\tau^{-1}\left(x_{t}-x_{t-\tau}\right)-A \hat{x}_{t}+P \operatorname{sign}\left(\Delta_{t}\right)\right]} \\
& \cdot\left[\begin{array}{c}
\sigma\left(\hat{x}_{t}\right) \\
\phi\left(\hat{x}_{t}\right) u_{t}
\end{array}\right]^{+}
\end{aligned}
$$

where $[\cdot]^{+}$stands in the Moore-Penrose sense [1]. This learning law is just an algebraic relation depending on $\Delta_{t}$, which can be evaluated directly. Taking into account that $x^{+}=\left(x^{T} /\|x\|^{2}\right)$, the last formula (12) can be rewritten as follows:

$$
\begin{aligned}
{\left[\hat{W}_{1, t} \quad \hat{W}_{2, t}\right]=} & \frac{\left[\tau^{-1}\left(x_{t}-x_{t-\tau}\right)-A \hat{x}_{t}+P \operatorname{sign}\left(\Delta_{t}\right)\right]}{\left\|\sigma\left(\hat{x}_{t}\right)\right\|^{2}+\left\|\phi\left(\hat{x}_{t}\right) u_{t}\right\|^{2}} \\
& \cdot\left[\begin{array}{c}
\sigma\left(\hat{x}_{t}\right) \\
\phi\left(\hat{x}_{t}\right) u_{t}
\end{array}\right]^{T} .
\end{aligned}
$$

To analyze the stability of the estimation error, we define the Lyapunov function as $V_{t}=\frac{1}{2}\left\|\Delta_{t}\right\|^{2}$. Calculating its derivative along the trajectories of the differential equations (9) and (10) we get

$$
\begin{aligned}
\dot{V}_{t} & =\Delta_{t}^{T} \dot{\Delta}_{t}=\Delta_{t}^{T}\left(-P \operatorname{sign}\left(\Delta_{t}\right)+\delta_{t}\right) \\
& =-\sum_{i=1}^{n} P_{i}\left|\Delta_{i}\right|+\Delta_{t}^{T} \delta_{t} \\
& \leq-\min _{i} P_{i}\left\|\Delta_{t}\right\|+\left\|\Delta_{t}\right\|\left\|\delta_{t}\right\| .
\end{aligned}
$$

Applying the bounds given by (8), we obtain

$$
\dot{V}_{t} \leq-\left\|\Delta_{t}\right\|\left[\min _{i} P_{i}-\left(C_{\tau}+\frac{\tau}{2} D_{\tau}\right)\right] .
$$

If we select a big enough $P_{i}$ as

$$
\min _{i} P_{i}>C_{\tau}+\frac{\tau}{2} D_{\tau}
$$

then $\dot{V}_{t}<0$ and we can guarantee the property $\Delta_{t} \rightarrow 0$. To realize (14), it is desirable to select $\tau$ as small as possible.

\section{Local Optimal Control via the Neural IDENTifier}

Given an unknown chaotic system, the control aim is to force it to set points or a stable trajectory. Because we cannot design a direct controller based on the chaotic model, an identifier-based controller is used. Let us assume that the desired tracking trajectory is a solution of a known nonlinear reference model given by

$$
\dot{x}^{*}=\varphi\left(x^{*}, t\right), \quad x_{t}^{*} \in \Re^{n}, \quad x^{*}(0)=c
$$

where $c$ is a constant. In the case of the regulation problem, $\varphi\left(x_{t}^{*}, t\right)=0$. The nonlinear system (1) can be represented as the neural network (5), plus a modeling error $\Delta f$

$$
f\left(x_{t}, u_{t}, t\right)=A x+W_{1, t} \sigma(x)+W_{2, t} \phi(x) u_{t}+\Delta . f
$$

The nonlinear system (1) may be represented as

$$
\begin{aligned}
\dot{x}_{t}= & A x_{t}+W_{1, t} \sigma\left(\hat{x}_{t}\right)+W_{2, t} \phi\left(\hat{x}_{t}\right) u_{t}+\Delta f \\
& +W_{1, t} \tilde{\sigma}+W_{2, t} \tilde{\phi} u_{t} \\
= & A x_{t}+W_{1, t} \sigma\left(\hat{x}_{t}\right)+W_{2, t} \phi\left(\hat{x}_{t}\right) u_{t}+d_{t}
\end{aligned}
$$

where $\tilde{\sigma}:=\sigma\left(x_{t}\right)-\sigma\left(\hat{x}_{t}\right), \tilde{\phi}_{t}:=\phi\left(x_{t}\right)-\phi\left(\hat{x}_{t}\right), d_{t}=\Delta f+W_{1, t} \tilde{\sigma}+$ $W_{2, t} \tilde{\phi} u_{t}$. Defining the trajectory error $\Delta_{t}^{*}=x_{t}-x_{t}^{*}$ we get

$$
\dot{\Delta}_{t}^{*}=A x_{t}+W_{1, t} \sigma\left(\hat{x}_{t}\right)+W_{2, t} \phi\left(\hat{x}_{t}\right) u_{t}+d_{t}-\varphi\left(x_{t}^{*}, t\right) .
$$

We select the control action $u_{t}$ as (see [4])

$$
u_{t}=u_{1, t}+\left[W_{2, t} \phi\left(\hat{x}_{t}\right)\right]^{-1} u_{2, t}
$$


where $u_{1, t} \in \Re^{n}$ is direct control part and $u_{2, t} \in \Re^{n}$ is a compensation of unmodeled dynamic $d_{t}$. Because $\varphi\left(x_{t}^{*}, t\right), x_{t}^{*}$, $W_{1, t} \sigma\left(\hat{x}_{t}\right)$ and $W_{2, t} \phi\left(\hat{x}_{t}\right)$ are available, we can select $u_{1, t}$ as

$$
u_{1, t}=\left[W_{2, t} \phi\left(\hat{x}_{t}\right)\right]^{-1}\left[\varphi\left(x_{t}^{*}, t\right)-A x_{t}^{*}-W_{1, t} \sigma\left(\hat{x}_{t}\right)\right] .
$$

Therefore, (17) becomes $\dot{\Delta}_{t}^{*}=A \Delta_{t}^{*}+u_{2, t}+d_{t}$. A local optimal (minimal variance) control [4] may be applied to compensate the unmodeled dynamics. We may select stable matrixes $A, R$ and $Q_{c}$ to satisfy the following conditions. The pair $\left(A, R^{1 / 2}\right)$ is controllable, the pair $\left(Q_{c}^{1 / 2}, A\right)$ is observable, and a special local frequency condition [11] fulfills, which turns out to be equal to the matrix inequality

$$
\begin{aligned}
A^{T} R^{-1} A-Q_{c} \geq & \frac{1}{4}\left[A^{T} R^{-1}-R^{-1} A\right] \\
& \cdot R\left[A^{T} R^{-1}-R^{-1} A\right]^{T} \geq 0 .
\end{aligned}
$$

Thus, all of these conditions are equal to the following assumption [11].

A2: There exist positive definite matrixes $A$ and $Q_{c}$ such that the matrix Riccati equation

$$
A^{T} P_{c}+P_{c} A+P_{c} R P_{c}+Q_{c}=0
$$

has positive solution $P_{c}$.

Theorem 1: For the neural network (5) subjected to A1 and A2, the given system (1), and the reference model (15), the tracking error $\Delta_{t}^{*}$ satisfies

$$
\begin{aligned}
\varlimsup_{\tau \rightarrow \infty} & \frac{1}{\tau} \int_{0}^{\tau}\left[\Delta_{t}^{* T} Q_{c} \Delta_{t}^{*}+u_{2, t} R_{c} u_{2, t}\right] d t \\
\leq & \varlimsup_{\tau \rightarrow \infty} \frac{1}{\tau} \int_{0}^{\tau}\left[d_{t}^{T} R^{-1} d_{t}+\Psi\left(u_{2, t}\right)\right] d t
\end{aligned}
$$

where $R_{c}$ is given positive define matrix and $\Psi\left(u_{2, t}\right)=$ $2 \Delta_{t}^{* T} P_{c} u_{2, t}+u_{2, t}^{T} R_{c} u_{2, t}$.

Proof: In order to analyze the tracking error stability, we introduce the following Lyapunov function: $V_{t}^{*}=\Delta_{t}^{* T} P_{c} \Delta_{t}^{*}, P_{c}=$ $P_{c}>0$, whose time derivative is equal to

$$
\dot{V}_{t}^{*}=\Delta_{t}^{* T}\left(A^{T} P_{c}+P_{c} A\right) \Delta_{t}^{*}+2 \Delta_{t}^{* T} P_{c} u_{2, t}+2 \Delta_{t}^{* T} P_{c} d_{t} .
$$

Using the matrix inequality [6]

$$
X^{T} Y+Y^{T} X \leq X^{T} \Lambda X+Y^{T} \Lambda^{-1} Y
$$

which is valid for any matrixes $X, Y \in R^{n \times m}$ and any $0<\Lambda=$ $\Lambda^{T} \in R^{n \times n}$, the term $2 \Delta_{t}^{* T} P_{c} d_{t}$ can be estimated as

$$
2 \Delta_{t}^{* T} P_{c} d_{t} \leq \Delta_{t}^{* T} P_{c} R^{-1} P_{c} \Delta_{t}^{*}+d_{t}^{T} R d_{t} .
$$

Substituting (24) in (23) and adding and subtracting the term $\Delta_{t}^{* T} Q_{c} \Delta_{t}^{*}$ and $\Delta u_{2, t}^{T} R_{c} u_{2, t}$, we formulate

$$
\begin{aligned}
\dot{V}_{t}^{*} \leq & \Delta_{t}^{T}\left(A^{T} P_{c}+P_{c} A+P_{c} R P_{c}+Q_{c}\right) \Delta_{t} \\
& +2 \Delta_{t}^{* T} P_{c} u_{2, t}+u_{2, t}^{T} R_{c} u_{2, t}+d_{t}^{T} \Lambda^{-1} d_{t} \\
& -\Delta_{t}^{* T} Q_{c} \Delta_{t}^{*}-u_{2, t}^{T} R_{c} u_{2, t} .
\end{aligned}
$$

Because of $\mathbf{A 2}$, the first term of (25) is equal to zero. Thus, (25) is

$$
\left\|\Delta_{t}\right\|_{Q_{c}}^{2}+\left\|u_{2, t}\right\|_{R_{c}}^{2} \leq \Psi\left(u_{2, t}\right)+d_{t}^{T} R^{-1} d_{t}-\dot{V}_{t}^{*}\left(\Delta_{t}\right) .
$$

Then, integrating each term from 0 to $\tau$ and dividing each term by $\tau$, we obtain

$$
\begin{aligned}
\varlimsup_{\tau \rightarrow \infty} & \frac{1}{\tau} \int_{0}^{\tau} \Delta_{t}^{T} Q_{c} \Delta_{t} d t+\varlimsup_{\tau \rightarrow \infty} \frac{1}{\tau} \int_{0}^{\tau} u_{2, t}^{T} R_{c} u_{2, t} d t \\
\leq & \varlimsup_{\tau \rightarrow \infty} \frac{1}{\tau} \int_{0}^{\tau} d_{t}^{T} R^{-1} d_{t} d t+\varlimsup_{\tau \rightarrow \infty} \frac{1}{\tau} \int_{0}^{\tau} \Psi\left(u_{2, t}\right) d t \\
& +\varlimsup_{\tau \rightarrow \infty}\left[-\frac{1}{\tau} \int_{0}^{\tau} \dot{V}_{t}^{*}\right] .
\end{aligned}
$$

Taking into account that $V_{t}^{*} \geq 0$, we conclude

$$
\begin{aligned}
\varlimsup_{\tau \rightarrow \infty}\left[-\frac{1}{\tau} \int_{0}^{\tau} \dot{V}_{t}^{*}\right] & =\varlimsup_{\tau \rightarrow \infty}\left[-\frac{1}{\tau} V_{t}^{*}+\frac{1}{\tau} V_{0}^{*}\right] \\
& \leq \varlimsup_{\tau \rightarrow \infty}\left[\frac{1}{\tau} V_{0}^{*}\right]=0 .
\end{aligned}
$$

Therefore, (22) is established. The theorem is proved.

The control goal now is to minimize $d_{t}^{T} R^{-1} d_{t}$ and $\Psi\left(u_{2, t}\right)$. To minimize $\left\|d_{t}\right\|_{R-1}^{2}$, we should minimize $R^{-1}$. From (20), if we select $A$ and $Q_{c}$ to provide a positive solution for (21), we must choose $R^{-1}=R_{\min }^{-1}=A^{-T} Q_{c} A^{-1}$. To minimize the energetic function $\Psi\left(u_{2}, t\right)$ at every time $t$ for the given $x_{t}^{*}$ and $\hat{x}_{t}$, which are assumed to be realized and not dependent on $u_{2, t}$, we should solve the following optimization problem as

$$
\left\{\begin{array}{l}
\min _{U_{2, t}} \Psi\left(u_{2, t}\right), \\
\text { subject: } \quad \alpha\left(u_{1, t}+u_{2, t}\right) \leq \beta
\end{array}\right.
$$

where $\alpha$ and $\beta$ are given matrixes reflecting the physical constraints. For example, in the case of the saturation type restriction, we have $\alpha=I$. This problem is a standard quadratic programming problem. We name the $u_{2, t}^{*}(t)$ as the locally optimal control [4], because it is calculated based only on local information $\left(x_{t}^{*}\right.$ and $\left.\hat{x}_{t}\right)$. In the case of no restrictions, $u_{2, t}$ can be found analytically and turns out to be the LQ-optimal control law (see [11])

$$
u_{2, t}^{*}=-2 R_{c}^{-1} P_{c} \Delta_{t}^{*} .
$$

\section{Simulation Results}

In this section, three typical chaotic system are chosen to demonstrate the abilities of the neuroidentifier and local optimal controller, based on these identification estimates.

(1) The Lorenz System: The Lorenz model is used for the fluid conviction description, especially for some feature of the atmospheric dynamic [10]. The uncontrolled model is given by

$$
\begin{aligned}
& \dot{x}_{1}=\sigma\left(x_{2}-x_{1}\right)+u_{1}, \\
& \dot{x}_{2}=\rho x_{1}-x_{2}-x_{1} x_{3}+u_{2}, \\
& \dot{x}_{3}=-\beta x_{3}+x_{1} x_{2}+u_{3}
\end{aligned}
$$

where $x_{1}, x_{2}$, and $x_{3}$ represent measures of fluid velocity and horizontal and vertical temperature variations, respectively. $\sigma, \rho$, and $\beta$ are positive parameters representing the Prandtl number, Rayleigh number, and geometric factor, respectively. If $\rho<1$, the origin is a stable equilibrium. If

$$
1<\rho \leq \rho^{*}(\sigma, \beta):=\sigma(\sigma+\beta+3) /(\sigma-\beta-1)
$$

the system has two stable equilibrium points $( \pm \sqrt{\beta(\rho-1)}$, $\pm \sqrt{\beta(\rho-1)},(\rho-1))$ and one unstable equilibrium (the origin). If $\rho^{*}(\sigma, \beta)<\rho$, all three equilibrium points become unstable. As in the the commonly studied case, we select $\sigma=10$ and $\beta=8 / 3$ which leads to $\rho^{*}(\sigma, \beta)=24.74$. In the next example we will consider the system with $\sigma=10, \beta=8 / 3$, and $\rho=28$. In order to construct a model-based controller, first, we design an online neuroidentifier as

$$
\dot{\hat{x}}_{t}=A \hat{x}_{t}+W_{1, t} \sigma\left(\hat{x}_{t}\right)+W_{2, t} \phi\left(\hat{x}_{t}\right) u_{t}
$$

where $A=\operatorname{diag}(-8,-8,-8)$. The initial conditions for $\hat{x}_{t}$ can be any small value. Here we select $\hat{x}_{0}=[1,-5,0]^{T}$. The weights $W_{1, t}$ and $W_{2, t}$ are $3 \times 3$ matrixes. The elements of $\phi(\cdot)$ and $\sigma(\cdot)$ are selected as $\sigma\left(x_{i}\right)=\left(2 / 1+e^{-2 x_{i}}\right)-0.5, \phi\left(x_{i}\right)=$ 


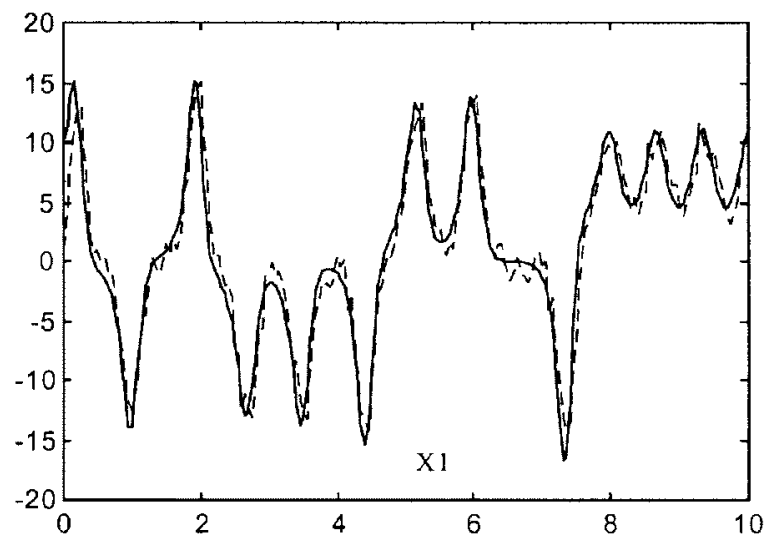

(a)

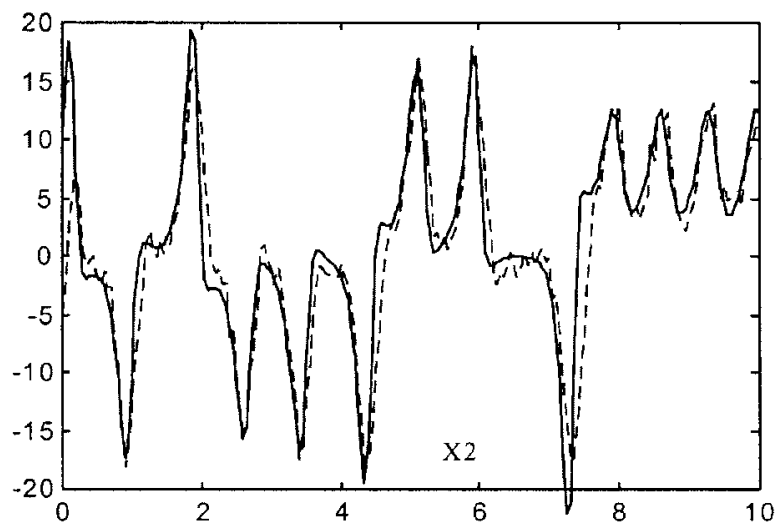

(b)

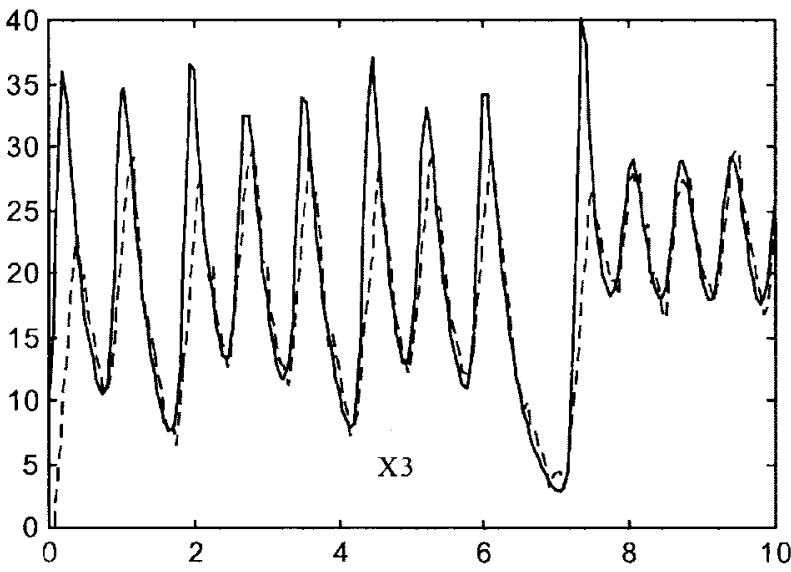

(c)

Fig. 1. (a) Identification results. (b) Identification results. (c) Identification results.

$\left(0.2 / 1+e^{-0.2 x_{i}}\right)-0.05 . P=\operatorname{diag}(20,20,20), \tau=0.01$. The identification results are shown in Fig. 1.

The solid lines correspond to the state of the uncontrolled Lorenz system and the dashed lines are the states of the neural network identifier. We observe that the average identification error is not more than ten. Because we adopt the sliding mode learning, this dynamic neural network can follow the fast system very well. We know that most of the existed updating laws of neural networks cannot give so quick a response. Therefore, based on those neuromodels, it is difficult to design an online controller which can guarantee a respectively good trajectory behavior. The drawback of these neuroidentifiers is that their weights change very quickly and, as a

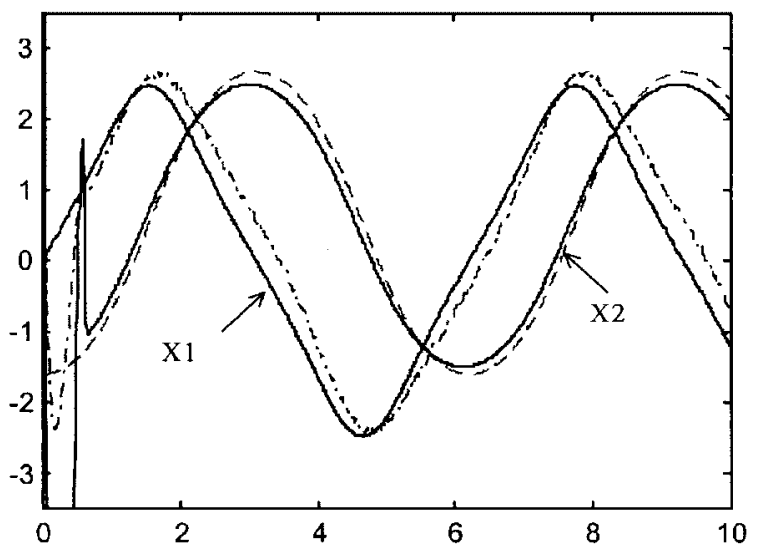

Fig. 2. Lorenz System.

result, it is not easy to use them in any control loop. If apply the derived sliding mode identifier to the local optimal controller, we can avoid these large deviations.

Then we implement the control law given by (19) and (26). The adaptive controller is based on the constructed neural network identifier. To simplify the computation, we assume the control input is free (it is not subjected to any restrictions). We select

$$
R_{C}=\left[\begin{array}{cc}
0.05 & 0 \\
0 & 0.05
\end{array}\right], \quad Q_{C}=I, \quad R=\left[\begin{array}{cc}
0.3 & 0 \\
0 & 0.3
\end{array}\right]
$$

the solution of the corresponding Riccati Equation is

$$
P_{c}=\left[\begin{array}{cc}
0.84 & 0 \\
0 & 0.84
\end{array}\right] \text {. }
$$

The nonlinear reference model to be followed is selected as a circle

$$
\dot{x}_{1}^{*}=x_{2}^{*}, \quad \dot{x}_{2}^{*}=\sin \left(x_{1}^{*}\right), \quad x_{3}^{*}=50
$$

with initial conditions equal to $x_{1}^{*}(0)=1, x_{2}^{*}(0)=0$. The trajectory tracking results are shown in Fig. 2. We observe that the average trajectory error does not exceed ten.

(2) The Duffing Equation: This describes a specific nonlinear circuit or the hardening spring effect observed in many mechanical problems [5] and can be written as

$$
\begin{aligned}
& \dot{x}_{1}=x_{2}, \\
& \dot{x}_{2}=-p_{1} x_{1}-p_{2} x_{1}^{3}-p x_{2}+q \cos (\omega t)+u_{t}
\end{aligned}
$$

where $p, p_{1}, p_{2}, q$, and $\omega$ are constants and $u_{t}$ is the control input. It is known that the solution of (30) exhibits almost periodic and chaotic behavior in the uncontrolled case if we select $p_{1}=1.1$, $p_{2}=1, p=0.4, q=2.1$, and $\omega=1.8$. Because the Duffing oscillator is a two dimensional (2-D) dynamic, to identify this system we use the same neural network as in (28), but with 2-D state space, i.e., $A=\operatorname{diag}(-8,-8), \hat{x}_{0}=[1,-5]^{T}, W_{1, t}$ and $W_{2, t}$ are $2 \times$ 2 matrixes. The elements of $\phi(\cdot)$ and $\sigma(\cdot)$ are selected as in (12) $P=\operatorname{diag}(20,20)$ and $\tau=0.01$. The controlled Duffing equation differs from the Lorenz system because it has only one control input. We also force the Duffing equation to the periodic orbits, as in (29). The corresponding results are shown in Fig. 3. We note that the local optimal controller is independent of the chaotic systems, because this controller is based only on the neuroidentifier. Numerical simulations show that good identification results provide a small enough tracking error. 


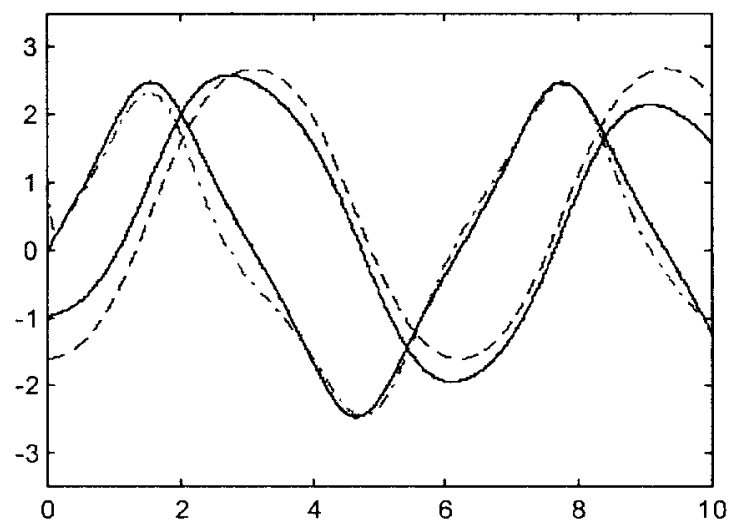

Fig. 3. Duffing Equation.

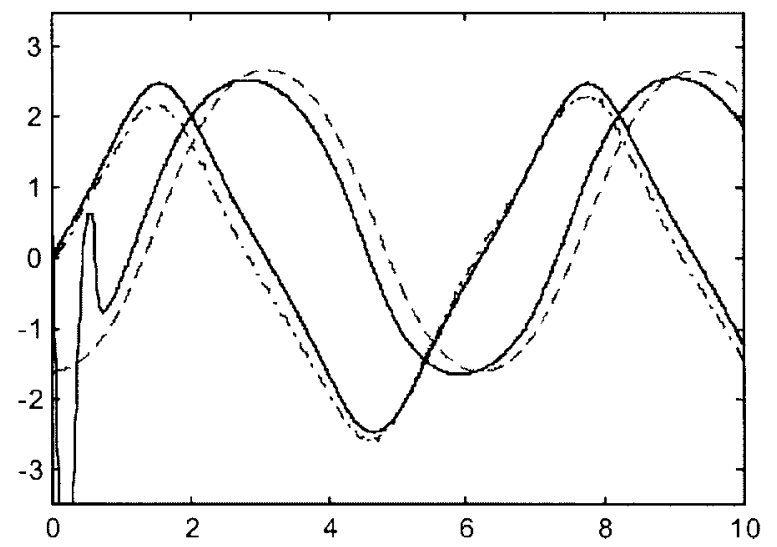

Fig. 4. Chua's Circuit.

(3) Chua's Circuit: This is an interesting electronics system that displays rich and typical bifurcation and chaotic phenomena, such as double scroll and double hook [2]. To study the controlled circuit, we formulate its equation in the following form:

$$
\begin{aligned}
& C_{1} \dot{x}_{1}=G\left(x_{2}-x_{1}\right)-g\left(x_{1}\right)+u_{1} \\
& C_{2} \dot{x}_{2}=G\left(x_{1}-x_{2}\right)+x_{3}+u_{2}, \quad L \dot{x}_{3}=-x_{2},
\end{aligned}
$$

where $g\left(x_{1}\right)=m_{0} x_{1}+\frac{1}{2}\left(m_{1}-m_{0}\right)\left[\left|x_{1}+B_{p}\right|+\left|x_{1}-B_{p}\right|\right], x_{1}$, $x_{2}, x_{3}$ denote, respectively, the voltages across $C_{1}$ and $C_{2}$ and the current through $L$. It is known that with $\left(1 / C_{1}\right)=9,\left(1 / C_{2}\right)=1$, $(1 / L)=7, G=0.7, m_{0}=-\frac{1}{2}, m_{1}=-\frac{4}{5}, B_{p}=1$, the circuit displays a double scroll. To demonstrate the effectiveness of the approach suggested in this study, we also use the same neural network as in (28). The controlled tracking behavior is shown in Fig. 4.

\section{CONCLUSION}

We present a new control method for unknown chaotic systems. The identifier uses the sliding mode technique to increase the learning speed of neural network weights. This approach can be considered as an alternative to LSM to avoid the recurrent/differential backpropagation learning. Based on the identifier, the local optimal control is used. Simulation results show that for different chaos under different initial conditions, the derived control via the neuroidentifier turns out to be very effective.

\section{REFERENCES}

[1] Regression and the Moore-Penrose Pseudoinverse. New York: Academic, 1972.

[2] G. Chen and X. Dong, "Identification and control of chaotic systems," in Proc. IEEE Int. Symp. Circuits Systems, Seattle, WA, 1995.

[3] J. A. Gallegos, "Nonlinear regulation of a Lorenz system by feedback linearization techniques," Dynamic Contr., vol. 4, 277-298, 1994.

[4] G. K. Kel'mans, A. S. Poznyak, and A. V. Chernitser, "Adaptive locally optimal control," Int. J. Syst. Sci., vol. 12, pp. 235-254, 1981.

[5] H. Nijmeijer and H. Berghuis, "On Lyapunov control of the Duffing equation," IEEE Trans. Circuits Syst., vol. 42, pp. 473-477, Aug. 1995.

[6] A. S. Poznyak, W. Yu, H. S. Ramirez, and E. N. Sanchez, "Robust identification by dynamic neural networks using sliding mode learning," App. Math. Comput. Sci. , vol. 8, 101-110, 1998.

[7] G. A. Rovithakis and M. A. Christodoulou, "Adaptive control of unknown plants using dynamical neural networks," IEEE Trans. Syst., Man Cybern., vol. 24, no. 3, pp 400-412, 1994.

[8] V. I. Utkin, Sliding Modes in Optimization and Control. Berlin, Germany: Springer-Verlag, 1992.

[9] T. H. Yeap and N. U. Ahmed, "Feedback control of chaotic systems," Dynamic Contr., vol. 4, 97-114, 1994.

[10] Y. Zeng and S. N. Singh, "Adaptive control cf Chaos in Lorenz system," Dynamic Contr., vol. 7, 143-154, 1997.

[11] J. C. Willems, "Least squares optimal control and algebraic Riccati equations," IEEE Trans. Automat. Contr., vol. 16, no. 6, 621-634, 1971.

\section{Direct Canonic Synthesis of All-Pass Digital Filter Structures}

\author{
T. Venkateswarlu
}

\begin{abstract}
A structure for realization of the $n$ th-order all-pass digital filter is presented. A known synthesis approach is used to obtain filter structures. The method is simple and the resulting structure contains $n$ multipliers and $n$ unit delay elements. These structures possess modularity and are, hence, attractive for VLSI implementation.
\end{abstract}

Index Terms - All-pass, digital filters, modularity, structure, VLSI, wave characterization.

A method for realizing $n$ th-order all-pass digital filters has been reported in [1]. This method uses the $z$-domain procedure for realizing all-pass digital filters and is similar to the methods reported in [2] and [3] for the multidimensional case. Another approach to realize digital filters is to use an analog reference configuration and then apply the wave characterization to obtain the corresponding digital filter structure.

It is known that the cascade digital filter structures, designed comprising generalized-immittance-converters (GIC's) and resistors, possess many advantages such low sensitivity, low noise and the absence of limit cycles characteristics, as compared to the direct form cascade structures [4]-[5]. The aim of this paper is to show that an alternate $n$ th-order all-pass digital filter can be obtained by using an analog reference configuration, comprising GIC's and resistors.

Manuscript received August 25, 1998. This paper was recommended by Associate Editor G. Martinelli.

The author is with the Faculty of Engineering, Universiti Telekom, Bukit Beruang, Jalan Ayer Keroh Lama, 75450 Melaka, Malaysia (e-mail: warlu@unitele.com.my).

Publisher Item Identifier S 1057-7122(99)09751-2. 